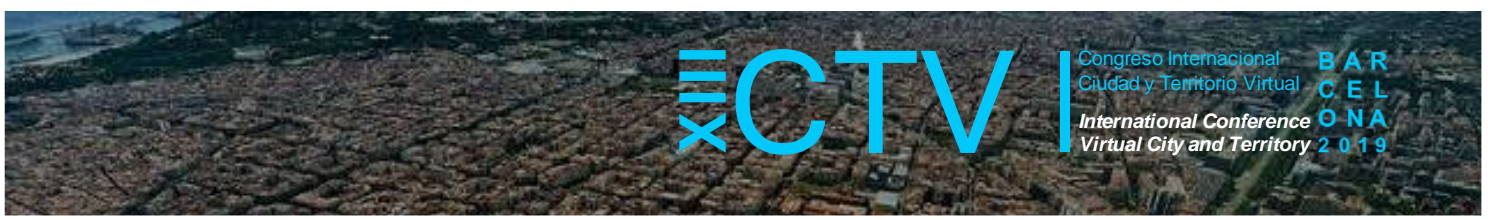

\title{
Elementos para el diagnóstico de la resiliencia por incremento en el nivel del mar en ciudades costeras del caribe colombiano
}

\author{
Niño Soto, Alexander Stward ${ }^{1 *}$; Hurtado Morales, Jim Héctor ${ }^{2}$
}

Remisión inicial: 2019-06-13; Remisión definitiva: 2019-10-03; Publicación: 2019-12-21

Citación: Niño, A. \& Hurtado, J. (2019). Elementos para el diagnóstico de la resiliencia por incremento en el nivel del mar en ciudades costeras del caribe colombiano. En XIII CTV 2019 Proceedings: XIII International Conference on Virtual City and Territory: "Challenges and paradigms of the contemporary city”: UPC, Barcelona, October 2-4, 2019. Barcelona: CPSV, 2019, p. 8541. E-ISSN 2604-6512. DOI http://dx.doi.org/10.5821/ctv.8541

\section{Resumen}

El objetivo general de la investigación exploraba construir un modelo de valoración de la resiliencia urbana, para ciudades costeras en la costa caribe de Colombia, en el cual se estudiaron los diferentes enfoques sobre la resiliencia urbana en poblados y ciudades costeras; a partir de la construcción de elementos medibles en los centros urbanos, que puedan ser utilizados como instrumento de planificación y ordenamiento territorial. La exploración se cumplió por etapas, que vinculó una primera fase de elaboración conceptual y metodológica a partir de la gestión del riesgo, para la construcción del conjunto de indicadores que aportaron información para la evaluación de la resiliencia en entornos urbanos costeros. Luego, se identificaron los principios de resiliencia urbana para la formulación de modelos de urbanismo sostenible en ciudades costeras. Así, se consultaron los estudios realizados por las autoridades estatales costeras y se cruzaron con las localizaciones de los centros urbanos. Luego, de la anterior construcción conceptual y metodológica, se procedió a proyectar cartográficamente los alcances de afectación por aumento en el nivel del mar, determinado por el incremento en la temperatura global, lo que permitió obtener datos para la elaboración de los perfiles cualitativo y cuantitativo de afectación sobre los centros urbanos costeros. Así, se estimó una valoración de impacto y la magnitud de respuesta de acuerdo con sus capacidades instaladas y cuantificadas, para ser referidas como indicador del nivel de resiliencia de cada sistema urbano. Se recolectó información a partir de los estudios y proyecciones de inundación realizados por Climate Central Organization, con disponibilidad abierta en la herramienta Surging Seas. A partir de las imágenes georreferenciadas de los modelos de Surging Seas e imágenes satelitales de Google Earth pro, sobre las que se superpone información planimétrica del Departamento Administrativo de Estadística - DANE de Colombia; con estos insumos se construyeron las cartografías de cada uno de los municipios costeros de las Unidades Ambientales Costeras del Darién, Golfo de Morrosquillo, Rio Magdalena, Sierra Nevada de Santa Marta y La Guajira. Se elaboraron un total de 39 mapas de 25 municipios y sus zonas de influencia, así mismo se construyeron tablas de análisis e interpretación de los datos, en los que se cuantificaron los impactos por aumento en el nivel del mar en tres escenarios de incremento en la temperatura global cada uno sobre cartografía modelada para cada una de las cabeceras municipales y se evaluaron indicadores relevantes para el diagnóstico de las dimensiones social, ambiental y económica, que con la implementación de métodos de inducción al riesgo, pudieran permitir la estimación de la capacidad de resistencia y respuesta al impacto inducido, traducido en el nivel de resiliencia de cada uno de los municipios estudiados. Los estudios realizados permitieron estimar que de la muestra analizada correspondiente a 264 $\mathrm{Km}^{2}$, el $52 \%$ sufrirá graves impactos con un incremento relativo en la temperatura global de $1,5^{\circ} \mathrm{C}$, es decir en el rango más conservador de aumento en el nivel del mar - ANM de acuerdo con los estudios de referencia, lo que puede derivar en 1'184.353 habitantes potencialmente afectados, además del severo impacto a un amplio porcentaje de todo tipo de infraestructura de soporte social, económico y de servicios ecosistémicos de los municipios analizados. Es evidente que el impacto generado por el incremento en los niveles del mar, proyectados de acuerdo con la expectativa de incremento de la temperatura global, dejará en muy críticas condiciones a los municipios costeros; pero esencialmente se quedará sin administración central los departamentos y las ciudades emblemáticas de la costa caribe colombiana. Razón por la cual, es inminente la generación de planes de evacuación, traslado y acomodación progresiva hacia el continente de la población con amenaza latente, restringiendo los futuros desarrollos en áreas con comprobación de amenazas y la generación de sistemas naturales de amortiguación del territorio, todo ello con el importante aporte de programas de investigación robustos y multidisciplinares que sustenten la toma de decisiones. Por

\footnotetext{
1 Arquitecto, MSc., Doctor en Ciudad, Territorio y Sustentabilidad. Profesor Universidad del Norte, Colombia, https://orcid.org/0000-0002-1725-2242; ${ }^{2}$ Arquitecto, estudiante maestría Urbanismo y Desarrollo Territorial Universidad del Norte, Colombia, https://orcid.org/0000-0001-7603-1856. "Correos de contacto: asnino@uninorte.edu.co, alexanderninosoto@yahoo.com
} 


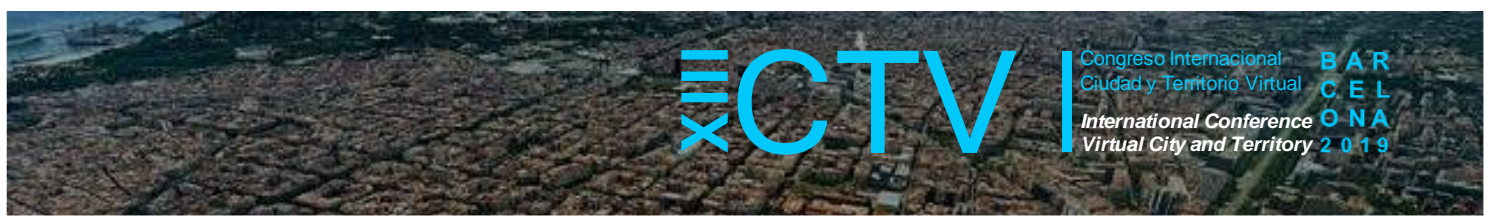

lo tanto, los planes de ordenamiento territorial deben empezar a actuar con la premisa de la real inundación como condición de planeación territorial.

\begin{abstract}
The general objective of the research was to build a model of urban resilience assessment, for coastal cities on the Caribbean coast of Colombia, in which different approaches to urban resilience in coastal towns and cities were studied; from the construction of measurable elements in urban centers, which can be used as an instrument of planning and territorial planning. The exploration was carried out in stages, which linked a first phase of conceptual and methodological elaboration based on risk management, for the construction of the set of indicators that provided information for the evaluation of resilience in coastal urban environments. Then, the principles of urban resilience were identified for the formulation of sustainable urban planning models in coastal cities. Thus, the studies carried out by the coastal state authorities were consulted and crossed with the locations of the urban centers. Then, from the previous conceptual and methodological construction, the scope of affectation due to increase in sea level was determined cartographically, determined by the increase in global temperature, which allowed obtaining data for the preparation of qualitative and quantitative profiles of impact on coastal urban centers. Thus, an impact assessment and the magnitude of response were estimated according to their installed and quantified capacities, to be referred to as an indicator of the level of resilience of each urban system. Information was collected from flood studies and projections conducted by the Climate Central Organization, with open availability in the Surging Seas tool. From the georeferenced images of the Surging Seas models and Google Earth pro satellite images, on which planimetric information from the Administrative Department of Statistics - DANE of Colombia is superimposed; With these inputs the cartographies of each of the coastal municipalities of the Coastal Environmental Units of Darién, Gulf of Morrosquillo, Rio Magdalena, Sierra Nevada de Santa Marta and La Guajira were built. A total of 39 maps of 25 municipalities and their areas of influence were prepared, as well as tables of analysis and interpretation of the data, in which the impacts due to sea level increase were quantified in three scenarios of increase in the global temperature each on modeled mapping for each of the municipal headings and relevant indicators were evaluated for the diagnosis of the social, environmental and economic dimensions, which with the implementation of risk induction methods, could allow the estimation of the capacity of resistance and response to induced impact, translated into the level of resilience of each of the municipalities studied. The studies made it possible to estimate that of the analyzed sample corresponding to $264 \mathrm{~km}^{2}, 52 \%$ will suffer serious impacts with a relative increase in the global temperature of $1.5^{\circ} \mathrm{C}$, that is, in the most conservative range of increase in sea level - ANM according to the reference studies, which can lead to $1,184,353$ potentially affected inhabitants, in addition to the severe impact on a large percentage of all types of social, economic and ecosystem services infrastructure of the municipalities analyzed. It is evident that the impact generated by the increase in sea levels, projected in accordance with the expectation of global temperature increase, will leave coastal municipalities in very critical conditions; But essentially the departments and emblematic cities of the Colombian Caribbean coast will run out of central administration. Reason why, the generation of evacuation, relocation and progressive accommodation plans towards the continent of the population with latent threat is imminent, restricting future developments in areas with threat testing and the generation of natural systems of buffering the territory, all This is done with the important contribution of robust and multidisciplinary research programs that support decision making. Therefore, land planning plans must begin to act on the premise of real flooding as a condition of territorial planning.
\end{abstract}

Palabras Clave: Sostenibilidad Urbana, Análisis Territorial, Cambio Climático

Key words: Urban Sustainability, Territorial Analysis, Climate Change

\title{
1. Introducción
}

El Grupo Intergubernamental de Expertos sobre el Cambio Climático - IPCC, publicó que debido al incremento en $1,5^{\circ} \mathrm{C}$ sobre la temperatura global, se presentan grandes impactos en los ecosistemas y en la sociedad (Naciones Unidas, 2019) por lo tanto, hay un gran riesgo para la región de América Latina y el Caribe cuyos asentamientos urbanos, en proceso de expansión, tienen cerca del $80 \%$ de su población en ciudades (División de Población de la Comisión Económica para América Latina y el Caribe-CEPAL - Centro Latinoamericano y Caribeño de Demografía-CELADE, 2018), lo que implica la necesidad de evaluar los impactos del cambio climático, medidos desde lo local y de manera integral a partir de la vulnerabilidad, la exposición y la susceptibilidad a los riegos del calentamiento global. 




Así, el crecimiento sin control de los asentamientos humanos, consecuencia del modelo económico (Rubiera Morollón, González Marroquin, \& Pérez Rivero, 2016), genera presión en los ecosistemas, por la imposición de las condiciones urbanas sobre un territorio; pues, expandir o densificar representa conflicto ambiental (Næss, Saglie, \& Richardson, 2019), evidenciando la vulnerabilidad y la improvisación de un sistema cambiante. Entonces, los centros urbanos son un sistema expansivo de ciclo abierto con demandas que ocasionan deterioro y degradación de los ecosistemas (Pedersen Zari, 2015). Entonces la fragilidad de los sistemas naturales por la inserción de patrones urbanísticos, origina el agotamiento de los recursos, aumenta la exposición ante eventos críticos e incrementa la susceptibilidad urbana ante acontecimientos de cambio climático.

En este contexto, es frecuente que los desastres se intensifiquen en asentamientos humanos mal planificados, pese a que todas las ciudades son susceptibles a eventos catastróficos (Rumbach, 2017), por esto las Naciones Unidas a través de la CEPAL y ONU HABITAT han promovido la planeación a partir de la evaluación del riesgo, la vulnerabilidad y la resiliencia. Así, con la Cumbre del Desarrollo Sostenible de 2015 se proclama la Agenda 2030, cuyo contenido expresa 17 Objetivos de Desarrollo Sostenible - ODS, que buscan generar planes y acciones, medibles y monitoreadas, en las diversas metas sobre la pobreza, la salud, la protección al ambiente y el manejo de los centros urbanos (Naciones Unidas, 2019). Así, en el marco del desarrollo globalizado y de la crisis del crecimiento, perfeccionar la resiliencia en el siglo XXI implica generar medidas para mayor cohesión social y comprensión ambiental sin importar la dimensión o protagonismo urbano (Méndez Gutiérrez del Valle, 2012). En este contexto, las ciudades del caribe colombiano deben brindar condiciones, pues falta formular estrategias de adaptación y resiliencia para la consolidación de asentamientos sostenibles, frente a los efectos del calentamiento global y al Aumento en el Nivel del Mar - ANM (Narula, 2014), puesto que los efectos directos por inundación marina impactarían fuertemente la población en centros poblados (Bedoya M. et al, 2010).

\section{Resiliencia Urbana}

La resiliencia es una capacidad que tienen los sistemas para tolerar distintas perturbaciones y recuperar su funcionamiento sin colapsar (Deal, Pan, Pallathucheril, \& Fulton, 2017). Entonces para los sistemas urbanos, la resiliencia se identifica como el resultado de una serie de procesos, que facilitan una habilidad adaptativa para retornar y mantener condiciones urbanas funcionales óptimas y flexibles ante los eventos (Meerow \& Newell, 2019) es decir, las medidas tendientes al mejoramiento de la sostenibilidad, la gobernabilidad, la mitigación de riegos, o la cohesión social, que han sido incorporadas en el marco de los ODS, son parte de la tendencia mundial para mejorar los niveles de resiliencia urbana (Leitner, Sheppard, Webber, \& Colven, 2018) por lo tanto, la resiliencia sería la habilidad para preparar un plan multidimensional que absorba, reconstruya y adapte los eventos adversos, previsibles o intempestivos y cuyas acciones sean la cultura de la resiliencia (Zhai, Li, \& Chen, 2015).

Entonces mejorar los niveles de resiliencia urbana, implica identificar las condiciones de vulnerabilidad ante eventos naturales vinculados a la exposición de los riesgos y peligros ambientales de los asentamientos. Dicha exposición, es la suma de complejidades multidimensionales, de situaciones generadas por los procesos de desarrollo económico y social, así como, de la explotación y uso de los ecosistemas para los modelos urbanos (Rumbach, 2017) Por otra parte, los comportamientos políticos, económicos y sociales son 


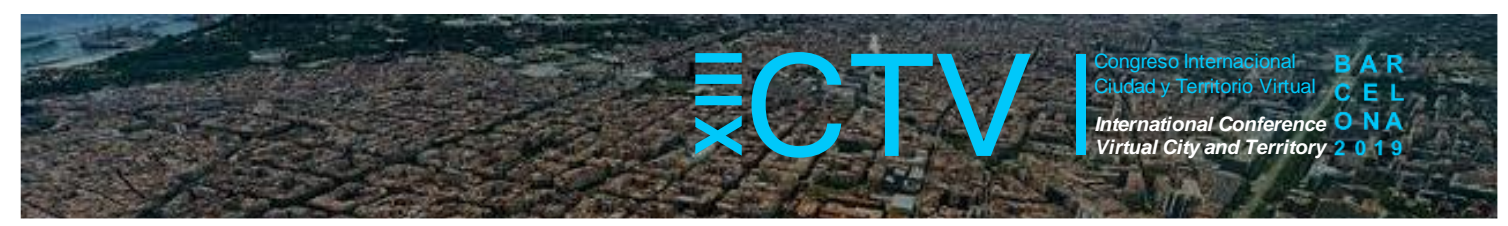

factores que influencian la capacidad resiliente, puesto que estas decisiones afectan la recuperación del sistema. Así, recobrar equilibrio conlleva una rápida y flexible capacidad de respuesta o una planificación adaptativa de recuperación. También, aumentar la capacidad de resiliencia requiere de ajustes y respuestas planeadas antes de la perturbación. Precisamente, la planeación demanda un sistema de respuesta proyectada bajo modelos predictivos como un conjunto de elementos y acciones de individuos y organizaciones, con los recursos e instrumentos para mitigar el daño y facilitar la capacidad de estabilización (Stead, 2014).

Tanto las ciudades compactas como las dispersas, generan demandas de recursos ambientales y energéticos sin límites de consumo en el territorio (Næss, Saglie, \& Richardson, 2019) no obstante, al modelo compacto se le atribuye que la ausencia de expansión urbana es un factor para mejorar la vulnerabilidad ante eventos. Pero, la presencia de desastres se encuentra asociada con la exposición del asentamiento ante un peligro mitigable o no, sin que sea compacto o disperso el modelo urbano. Entonces, el crecimiento urbano disperso aumenta la exposición y frecuencia a eventos de desastre que, en acontecimientos catastróficos, amplifican la evidencia de fracturas en los sistemas económicos, sociales y políticos de los centros urbanos (Rumbach, 2017) debido al desequilibrio, aleatoriedad y espontaneidad sin límites territoriales.

Efectivamente, existe una diferencia fundamental entre la resiliencia y el equilibrio, ya que un sistema puede estar en armonía, pero tener una baja capacidad resiliente y, por el contrario, una alta capacidad de ajuste no implica una relación de equilibrio, pues las fluctuaciones adaptativas a los cambios favorecen la persistencia del sistema urbano, pero dista de una realidad equilibrada. Así mismo, la capacidad de resiliencia no implica un retorno a las condiciones previas a la disrupción, pues la alteración cambia la trayectoria del sistema, lo que involucra una secuencia de eventos en una estructura para mejorar la capacidad resiliente, entonces, el sistema evoluciona. Luego, la resiliencia permite soportar agresiones externas y responder para mantener las condiciones funcionales, por lo tanto, fortalecer la capacidad de mitigación y la flexibilidad para la adaptación, es planear el territorio. En consecuencia, la mitigación como estrategia previa y la adaptación como prospectiva son fundamentos para la planeación territorial, tanto en escenarios de mitigación del riesgo como del cambio climático para fortalecer la resiliencia urbana (Stead, 2014). Así, el monitoreo de múltiples sistemas para identificar alteraciones, la preparación para la adaptación ante los potenciales cambios, la gobernabilidad sólida con respuesta sistémica, la comprensión de la magnitud y escala del evento, la identificación de opciones y el mantenimiento de soportes ante decisiones críticas, mejoran la resiliencia. (Deal, Pan, Pallathucheril, \& Fulton, 2017) También hay factores que se utilizan para determinar la capacidad resiliente como: las fortalezas económicas en la región, las prácticas y niveles educativas de los trabajadores, la cohesión y la fortaleza social. (Zhai, Li, \& Chen, 2015) En conclusión, la resiliencia urbana como proceso reflexivo busca la anticipación de los eventos perturbadores e incrementa las redes de soporte para la adaptabilidad, utilizando los medios para garantizar la cohesión social ante eventos catastróficos.

Justamente, la gestión de las ciudades resilientes es sistémica y territorial pues inicia desde el análisis y valoración de los recursos disponibles, hacia la identificación de los puntos críticos para propiciar sinergias público-privadas (Oficina de las Naciones Unidas para la Reducción del Riesgo de Desastres - UNISDR, 2012). Por lo tanto, un completo conocimiento de los recursos, aporta un asertivo diagnóstico de cada asentamiento urbano y facilita el desarrollo de 


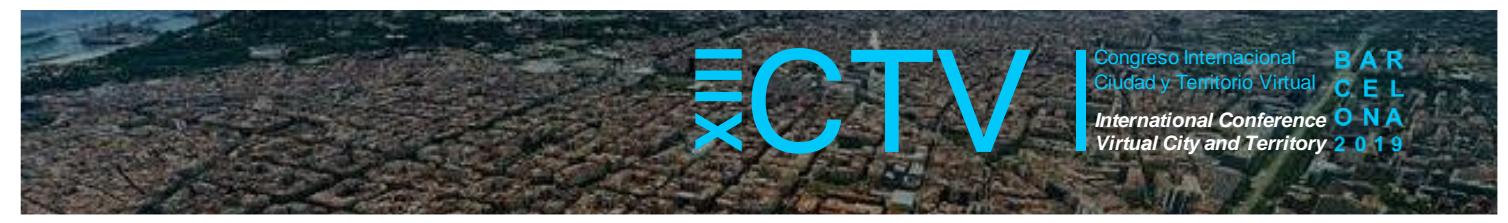

estrategias para ciudades resilientes, por consiguiente, aumenta las capacidades de restablecimiento (Oficina de las Naciones Unidas para la Reducción del Riesgo de Desastres (UNDRR), 2019). En este contexto, ONU HABITAT y ARUP GROUP han propuesto el desarrollo de herramientas para la elaboración de perfiles y diagnósticos de resiliencia, a partir de los componentes físicos del asentamiento, el capital ambiental, el componente social y los recursos económicos dispuestos para la atención de perturbaciones (UN-Habitat, 2018) (ARUP, 2011). También, ONU-Hábitat en el marco del programa City Resilience Profiling Programme (CRPP), busca proveer de métodos para la medición de la resiliencia y la identificación de estrategias frente al impacto de amenazas de múltiple índole con la herramienta City Resilience Profiling Tool - CRPT que parte del análisis de sistemas complejos, donde, infraestructura, medio ambiente, flujos y estructuras multinivel, interactúan dentro de un área urbana; permitiendo la identificación de aspectos funcionales y de soporte. (ONU HABITAT, 2018).

Para ello el CRPT plantea una metodología de diagnóstico participativo en materia de vulnerabilidad y fortalezas multisectoriales en distintas escalas, que valora atributos: espaciales, organizativos, físicos, funcionales y adaptativos mediante la aplicación de encuestas en 4 Sets. El Set 1, provee una visión general con datos contextuales, históricos, climáticos, ecosistémicos, urbanísticos y físicos; para otorgar identidad. Los SET 2, 3 y 4, aportan la profundidad del sistema urbano, pues vinculan personas, procesos y activos de la ciudad y evalúan interconexiones; construyendo un análisis de la funcionalidad del sistema urbano sobre la gobernanza con medidas y estrategias de reducción del riesgo de las amenazas. Finalmente, los datos son incorporados a un software produciendo el perfil de resiliencia para cada ciudad (UN-Habitat, 2018). Por otra parte, ARUP con el apoyo de la Fundación Rockefeller ha diseñado la herramienta The City Resilience Index, para monitoreo y medición de los múltiples factores en entornos urbanos, estructurada en cuatro dimensiones: salud y bienestar, economía y sociedad, infraestructura y medio ambiente, liderazgo y estrategia; 12 objetivos y 52 indicadores que contribuyen al desarrollo de ciudades resilientes. Su propósito es diagnosticar fortalezas y debilidades para medir el desempeño en el tiempo y proporcionar una base, que facilite el intercambio de conocimientos entre ciudades. El análisis del índice de resiliencia propuesto por Arup Group (2019) busca atributos para la configuración de respuestas efectivas ante la crisis, traducido como perfil o nivel de resiliencia. Finalmente, los resultados son catalogados por dimensión y forman la base para los planes de fortalecimiento sectorial. (Arup Group, 2019)

\section{La Resiliencia en la Costa Caribe de Colombia}

EL PNUD presenta 5 campos peligrosos del cambio climático: 1. La Producción agrícola y la seguridad alimentaria, 2. Los balances ecosistémicos y la seguridad de agua para los asentamientos humanos, 3. Deshielo y Aumento en el Nivel del Mar, 4. Ecosistemas y biodiversidad en riesgo; y 5. Riesgos a la Salud humana por calor y epidemias (Programa de la Naciones Unidas para el Desarrollo - PNUD, 2007). Estos riesgos proyectados sobre el $45 \%$ de la población mundial que vive en centros urbanos costeros, y la clara fragilidad de los sistemas urbanos en las regiones en vías de desarrollo (Oviedo Torres, 2010) (United Nations Development Programme - UNDP, 2014) aunados al escenario de elevación en el nivel del mar, en conjunto con el derretimiento de la criósfera y acompañado del mar extremo, incrementan los peligros en las ciudades costeras, es decir: la mitad de la población urbana mundial está en riesgo (Intergovernmental Panel on Climate Change - IPCC, 2019). Por lo 


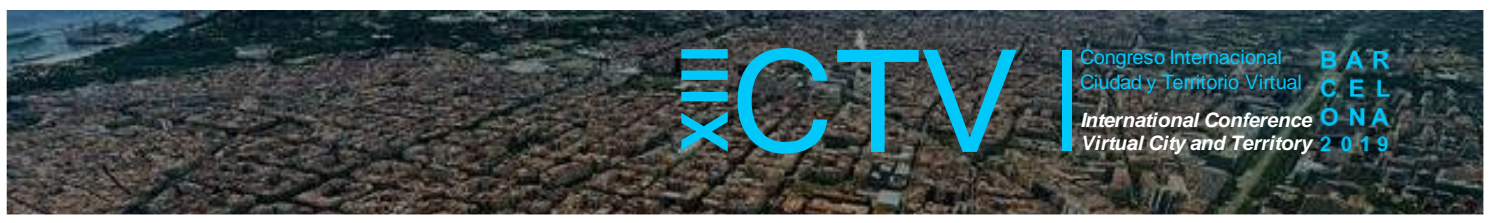

anterior, para Colombia en su costa caribe, el Cambio Climático y el Aumento en el Nivel de Mar - ANM, implican la medición de los potenciales impactos, así como la valoración de la capacidad resiliente de los centros urbanos. Para tal efecto, se planteó la elaboración de un perfil cuantitativo del riesgo frente al impacto del fenómeno ANM, teniendo como base la compilación, análisis y clasificación de información existente en fuentes documentales y un perfil cualitativo de respuesta que tiene como base el examen de indicadores agrupados en las dimensiones social, económica y ambiental.

\subsection{Perfil cuantitativo del riesgo}

Sobre los mapas de resolución en cuadrícula horizontal de aproximadamente 90 metros, producidos por la Misión Topográfica del Radar de la NASA (SRTM - Shuttle Radar Topography Mission) en el software Surging Seas, se observan elevaciones de superficie en tierra no desnuda, que presentan escenarios de afectación para los incrementos de $1,5^{\circ} \mathrm{C}$, $2,0^{\circ} \mathrm{C}$ y $3,0^{\circ} \mathrm{C}$ (CLIMATE CENTRAL, 2019). Los escenarios obtenidos sobre imágenes de Google Earth Pro en formato KML georefenciados, son contrastados y superpuestos con cartografía digital medible y escalada en formato DWG, provenientes del Geoportal del DANE (Departamento Administrativo Nacional de Estadística - DANE, 2019). Esto permitió graficar y cuantificar el área y los patrones de afectación sobre territorios específicos en cartografía georeferenciada en ArcGis, con tablas y gráficos estadísticos para el correspondiente perfil cuantitativo de afectación, valorando el impacto con los siguientes rangos de la Tabla 1:

Tabla 1. Rangos de Valoración

\begin{tabular}{llc}
\hline \multicolumn{1}{c}{ ESTADO } & \multicolumn{1}{c}{ VALORACIÓN } & PONDERACIÓN \\
\hline Condición Inviable & Afectación $\geq 80,1 \%$ de la muestra & 1 \\
\hline Riesgo Crítico & Afectación entre $60,1 \%$ y $80 \%$ de la muestra & 2 \\
\hline Atención Inmediata & Afectación entre 40,1\% y 60\% de la muestra & 3 \\
\hline Riesgo Bajo & Afectación entre 20,1\% y 40\% de la muestra & 4 \\
\hline Condición Viable & Afectación entre $0,1 \%$ y 20\% de la muestra & 5 \\
\hline
\end{tabular}

Fuente: construcción propia

\subsection{Perfil cualitativo de respuesta}

Se valoran las condiciones ecosistémicas, el estado y uso de los recursos ambientales; la accesibilidad y calidad de los servicios sobre condiciones de bienestar, así como la satisfacción de las necesidades básicas y del nivel de ingresos en función del poder adquisitivo de bienes y servicios. Así, se tomaron doce indicadores con información cuantitativa y cualitativa de la afectación sobre la muestra, asignando escalas de valor con respecto a su incidencia; agrupados en tres dimensiones: Ambiental, Social y Económica; partiendo de los criterios de pertinencia y afinidad para la asociación de los datos recolectados por municipios. La información de cada uno de los indicadores tiene como fuente la base datos del Sistema de Estadísticas Territoriales de Colombia - TERRIDATA (Departamento Nacional de Planeación DNP, 2019), Sistema de Información SISPRO (Ministerio de Salud y Protección Social, 2019), Sistema de Datos Transparencia por Colombia, Observatorio del Sistema de Ciudades (Departamento Nacional de Planeación - DNP, 2019) y el Sistema Estadístico Nacional (Departamento Administrativo Nacional de Estadística DANE, 2019), tomando como línea base de análisis diciembre de 2016. 


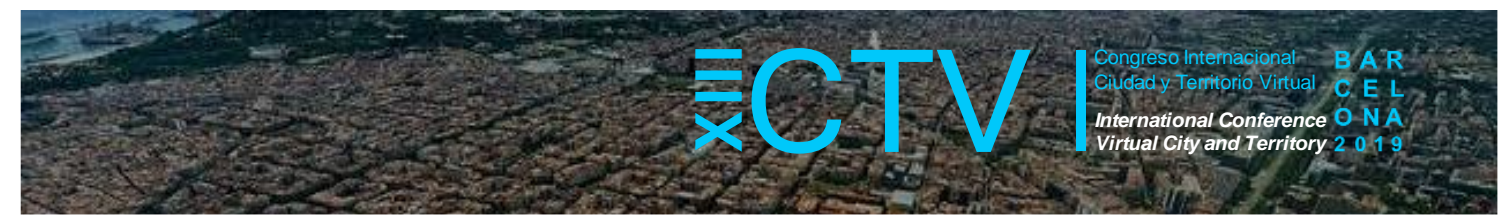

Sobre la Dimensión ambiental se identifica la calidad de los ecosistemas y su nivel de vulnerabilidad, el uso adecuado de los recursos naturales y las políticas de regulación ambiental; todo ello como indicador hacia una respuesta ambiental frente a la crisis. Esta dimensión se revisa a partir de los índices de 1. Riesgo ajustado por capacidades, 2. Vulnerabilidad y riesgo por cambio climático, 3. Área municipal de uso adecuado del suelo, y 4. Calidad del agua para consumo humano.

Así, el Índice de riesgo ajustado por capacidades se toma a partir del Índice Municipal de Riesgo de Desastres ajustado por Capacidades - IMRC (Departamento Nacional de Planeación - DNP, 2018) como una herramienta que mide el riesgo a nivel municipal ante eventos hidrometeorológicos asociados a aumentos de precipitaciones relacionado con las capacidades de las entidades territoriales para gestionarlo. El IMRC se construye a partir de los componentes del riesgo hacia la amenaza según áreas en condiciones críticas por inundación, remoción en masa y flujos torrenciales (IDEAM - Instituto de Hidrología, Meteorología y Estudios Ambientales, 2019) (Servicio Geológico de Colombia - SGC, 2019); y las capacidades se toman a partir de la exposición, pues corresponde a la proporción de la población del municipio expuesta a alguno de los tres fenómenos considerados en la amenaza. Así la vulnerabilidad, está referida a la predisposición sobre la afectación negativa por un evento físico peligroso. En el marco del Índice Municipal de Riesgo de Desastres, la vulnerabilidad refleja el grado de privación de las condiciones mínimas de calidad de vida de las personas del municipio, ya que para su medición fue empleado el índice sintético de condiciones de vida, elaborado por el DANE en el 2014. El índice de riesgo ajustado por capacidades, una vez se estandariza, asigna valores de cero (0) a cien (100), donde el cero está asociado a un municipio con menor riesgo y mayor capacidad, y cien, a un municipio con mayor riesgo y baja capacidad. Asimismo, se toma el índice de vulnerabilidad y riesgo por cambio climático que proviene de los riesgos de amenaza de desastres con los atributos de Análisis Multidimensional, Integración de análisis Marino costeros e insulares con análisis continentales, Comparación entre las unidades de análisis municipal y la generación de línea base con datos abiertos (IDEAM, PNUD, MADS, DNP, CANCILLERÍA, 2017). En cuanto al índice de porcentaje de área municipal en uso adecuado se utiliza la base de datos DNP (2019) que muestra al porcentaje del área municipal sobre la base de datos del Instituto Geográfico Agustín Codazzi. Para finalizar el índice de riesgo de la calidad del agua para consumo humano - IRCA, se toma desde la base de datos del Instituto Nacional de Salud (2007).

En cuanto a la dimensión social se relacionan los valores de bienestar, satisfacción de las necesidades básicas, regulación de la ley y el orden, accesibilidad a servicios públicos básicos y la educación como herramienta de fortalecimiento y crecimiento social; todo ello revisado a partir de 6 indicadores de respuesta social frente a la crisis. Entonces el Indicador de afectación a la vivienda, valora la proporción de hogares con carencias habitacionales sobre el total, para establecer el porcentaje de un déficit de cualidades de la Vivienda determinada. La cuantificación se obtiene con la sumatoria del déficit cuantitativo y cualitativo (DANE, 2009). Para el Indicador de proporción de personas con Necesidades Básicas Insatisfechas - NBI se toma el Boletín General del Censo 2005 del DANE que presenta la proporción de personas con NBI por sectores. En el caso del indicador de cobertura en relación con el porcentaje de oferta de servicios públicos básicos, también se consulta el Censo 2005, para la proporción de hogares con acceso a servicios públicos básicos de acueducto, alcantarillado, electricidad y gas. Sobre el indicador de educación se consulta las bases del Mineducación, de cobertura de estudiantes matriculados sobre el total de la población proyectada correspondiente. 


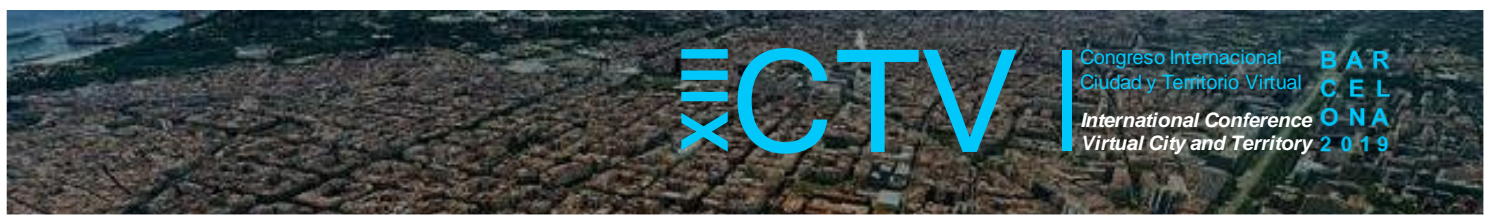

(MINISTERIO DE EDUCACIÓN NACIONAL, 2014) Para salud se toma el valor entre la relación del número de personas afiliadas al sistema de salud con respecto al total de la población estimada por el DANE (Ministerio de Salud y Protección Social, 2019). Al final el indicador de transparencia es tomado del Índice de Transparencia de las Entidades Públicas - ITEP, que se utiliza para medir el riesgo de corrupción administrativa (Córdoba Larrarte, Ocampo Niño, \& Villalba, 2015).

Para la dimensión económica, se revisan los indicadores de cobertura de ocupación, medidos por medio de la proporción de personas contratadas (población ocupada - OC) con respecto a la población total en edad de trabajar PET a partir de información del DNP. Y el indicador de cobertura de ingresos corrientes per cápita PIB pc que mide la relación existente entre el nivel de renta de un país y su población. Para ello, se divide el Producto Interior Bruto - PIB de dicho territorio entre el número de habitantes según valores del DANE.

Finalmente, los indicadores fueron condensados en tablas para mostrar los resultados en cada municipio que conforma las Unidades Ambientales Costeras - UAC, lo que representa la afectación directa por el fenómeno de Aumento en el Nivel del Mar - ANM. Para efecto del análisis de cada Unidad Ambiental Costera - UAC en particular, se tomaron los promedios de cada indicador. Por lo tanto, el análisis de afectación se realizó tomando el área urbana de cada municipio como objeto de impacto, partiendo del supuesto que los centros poblados reciben el mayor impacto en vivienda, movilidad, servicios y toda infraestructura de soporte sensible de deterioro y correspondiente reconstrucción. Así las afectaciones reflejadas por incremento en la temperatura de $1,5^{\circ} \mathrm{C} ; 2,0^{\circ} \mathrm{C}$ y $3,0^{\circ} \mathrm{C}$ fueron estimadas sobre el área urbana costera, como se puede observar en la tabla 2.

Tabla 2. modelo de estimación de inundación por incremento en la temperatura global

\begin{tabular}{|c|c|c|c|c|c|}
\hline \multirow{2}{*}{ AC } & \multirow{2}{*}{ CENTRO POBLADO } & \multirow{2}{*}{ AREA URBANA DANE km2 } & \multicolumn{3}{|c|}{ \% AFECTACIÓN (ANM) } \\
\hline & & & $1.5^{\circ}$ & $2.0^{\circ}$ & $3.0^{\circ}$ \\
\hline \multirow{9}{*}{ DARIEN } & Acandí & 0,7712 & $41,538 \%$ & $135,928 \%$ & $155,127 \%$ \\
\hline & Unguía & 1,1982 & $0,000 \%$ & $0,000 \%$ & $3,000 \%$ \\
\hline & Arboletes & 1,0086 & $26,978 \%$ & $38,466 \%$ & $71,913 \%$ \\
\hline & Turbo & 6,3813 & $83,880 \%$ & $171,540 \%$ & $174,712 \%$ \\
\hline & Necoclí & 2,4404 & $70,382 \%$ & $106,400 \%$ & $145,161 \%$ \\
\hline & San juán de urabá & \multirow{4}{*}{2,7393} & \multirow{4}{*}{$41,413 \%$} & \multirow{4}{*}{$85,688 \%$} & \multirow{4}{*}{$85,688 \%$} \\
\hline & Vda. Zapata & & & & \\
\hline & Vda. Damaquiel & & & & \\
\hline & Vda. Uveros & & & & \\
\hline \multirow{13}{*}{$\begin{array}{l}\text { GOLFO DE } \\
\text { MORROSQUILLO }\end{array}$} & Moñitos & \multirow{4}{*}{1,4126} & \multirow{4}{*}{$121,447 \%$} & \multirow{4}{*}{$239,288 \%$} & \multirow{4}{*}{$288,948 \%$} \\
\hline & Santander de la cruz & & & & \\
\hline & Broqueles & & & & \\
\hline & El cedro & & & & \\
\hline & Puerto escondido & \multirow{2}{*}{2,6283} & \multirow{2}{*}{$34,522 \%$} & \multirow{2}{*}{$100,000 \%$} & \multirow{2}{*}{$100,000 \%$} \\
\hline & Vda. Cristo rey & & & & \\
\hline & San antero & 4,1376 & $85,384 \%$ & $116,180 \%$ & $127,478 \%$ \\
\hline & San Bernardo del viento & 2,2347 & $37,799 \%$ & $37,799 \%$ & $291,792 \%$ \\
\hline & Santiago de tolú & 5,7218 & $75,115 \%$ & $144,339 \%$ & $190,121 \%$ \\
\hline & Coveñas & 8,3022 & $45,920 \%$ & $81,767 \%$ & $105,587 \%$ \\
\hline & San Onofre & \multirow{3}{*}{3,4675} & \multirow{3}{*}{$25,855 \%$} & \multirow{3}{*}{$67,421 \%$} & \multirow{3}{*}{$68,093 \%$} \\
\hline & Berrugas & & & & \\
\hline & Rincón del mar & & & & \\
\hline \multirow{9}{*}{ RIO MAGDALENA } & Galerazamba & \multirow{3}{*}{1,176} & \multirow{3}{*}{$97,948 \%$} & \multirow{3}{*}{$260,945 \%$} & \multirow{3}{*}{$348,999 \%$} \\
\hline & Loma de arena & & & & \\
\hline & Pueblo nuevo & & & & \\
\hline & Pasacaballos & \multirow{4}{*}{64,9152} & \multirow{4}{*}{$77,761 \%$} & \multirow{4}{*}{$106,174 \%$} & \\
\hline & Cartagena & & & & \\
\hline & La boquilla & & & & $115,066 \%$ \\
\hline & Manzanillo & & & & \\
\hline & Playa mendoza & 0,9698 & $21,083 \%$ & $31,579 \%$ & $36,141 \%$ \\
\hline & Puerto colombia & 5,1293 & $45,133 \%$ & $61,137 \%$ & $80,010 \%$ \\
\hline
\end{tabular}






\begin{tabular}{|c|c|c|c|c|c|}
\hline & Puerto Salgar & & & & \\
\hline & Santa Verónica & 2,434 & $0,000 \%$ & $0,000 \%$ & $0,000 \%$ \\
\hline & Barranquilla & 81,5704 & $21,196 \%$ & $54,172 \%$ & $59,813 \%$ \\
\hline & Ciénaga & 7,7884 & $100,195 \%$ & $168,212 \%$ & $198,668 \%$ \\
\hline \multirow{8}{*}{$\begin{array}{l}\text { SIERRA NEVADA } \\
\text { DE SANTA MARTA }\end{array}$} & Santa Marta & \multirow{3}{*}{34,173} & \multirow{3}{*}{$20,541 \%$} & \multirow{3}{*}{$29,271 \%$} & \multirow{3}{*}{$35,509 \%$} \\
\hline & Buritaca & & & & \\
\hline & Taganga & & & & \\
\hline & Riohacha & \multirow{2}{*}{13,5518} & \multirow{2}{*}{$51,876 \%$} & \multirow{2}{*}{$87,769 \%$} & \multirow{2}{*}{$131,436 \%$} \\
\hline & Camarones & & & & \\
\hline & Dibulla & \multirow{3}{*}{1,6884} & \multirow{3}{*}{$43,306 \%$} & \multirow{3}{*}{$55,538 \%$} & \multirow{3}{*}{$69,853 \%$} \\
\hline & La punta de los remedios & & & & \\
\hline & Palomino & & & & \\
\hline \multirow{3}{*}{ LA GUAJIRA } & Cabo de la vela & \multirow{2}{*}{4,0214} & \multirow{2}{*}{$9,353 \%$} & \multirow{2}{*}{$9,353 \%$} & \multirow{2}{*}{$9,353 \%$} \\
\hline & Uribia & & & & \\
\hline & Manaure & 4,0805 & $120,081 \%$ & $131,979 \%$ & $143,892 \%$ \\
\hline
\end{tabular}

Fuente: elaboración Propia

\subsection{Resultados}

Cerca del $36 \%$ del litoral caribe colombiano presentará afectación entre los rangos de condición inviable y riesgo crítico y un $16 \%$ requiere acciones inmediatas, con un incremento en la temperatura global del $1.5^{\circ} \mathrm{C}$, siendo los municipios de Turbo - Antioquia, Moñitos - Córdoba, San Antero - Córdoba, Santa Catalina - Bolívar, Ciénaga - Magdalena y Manaure - La Guajira, los casos más extremos. Es relevante anotar que, con un incremento de $0,5^{\circ} \mathrm{C}$ adicionales, los niveles de afectación pueden llegar a duplicarse, alcanzando al $64 \%$ de afectación entre los rangos de condición inviable y riesgo crítico y un $8 \%$ con requerimientos de atención inmediata. Es claro entonces que las poblaciones con impacto superior al $50 \%$ de su área urbana para un incremento de $1,5^{\circ} \mathrm{C}$ deben tomar medidas inmediatas.

A modo de detalles, en la UAC 1-Darién el $80 \%$ de los municipios y centros poblados, presentan afectación superior al $50 \%$ del área urbana con un incremento en la temperatura global de $1,5^{\circ} \mathrm{C}$ y afectación total para un incremento de $2,0^{\circ} \mathrm{C}$; el municipio de Unguía en el departamento del Chocó presenta impacto bajo, representado en la inundación de un 3\% del área urbana para un incremento en la temperatura de $3,0^{\circ} \mathrm{C}$. Para la UAC 2-Estuarina del río Sinú y el Golfo de Morrosquillo la mayor afectación está en los municipios de Coveñas y Santiago de Tolú en el departamento de Sucre y San Antero en el departamento de Córdoba; para los cuales un incremento de $1,5^{\circ} \mathrm{C}$ en la temperatura global, representaría la inundación del $50 \%$ del área urbana aproximadamente. Para los corregimientos de Cristo Rey, Rincón del Mar, El Cedro, Broqueles, Santander de la Cruz y Moñitos, el impacto resulta ser bastante drástico, teniendo en cuenta que la totalidad del área urbanizada quedaría inundada en el escenario de un incremento del $1,5^{\circ} \mathrm{C}$. El perfil cualitativo de la UAC Golfo de Morrosquillo expone un sistema con gran fragilidad en la gestión del componente ambiental y territorial, lo que sin duda restringe los recursos y la flexibilidad de los mecanismos de respuesta ante cualquier amenaza; la fragilidad descrita es potenciada por condiciones socio económicas precarias, que imposibilitan la estructuración de sistemas y planes de atención y prevención de desastres.

En el caso de la UAC 3-Río Magdalena, el modelo de inundación evidencia afectación del 36\% del total de los centros poblados con un incremento de $1,5^{\circ} \mathrm{C}$. Además del alto índice de erosión costera, los mayores riesgos de impacto a la infraestructura se dan lugar en el distrito de Cartagena, el municipio de Pasacaballos y la refinería. En afectación ecosistémica, la desaparición total de la línea de costa actual y la salinización del acuífero Ciénaga de Tesca, además del riesgo por reflujo sanitario al aumentar la presión sobre el emisor marítimo. Para Barranquilla y el municipio de Ciénaga, el incremento de $1,5^{\circ} \mathrm{C}$, representaría daños a los 


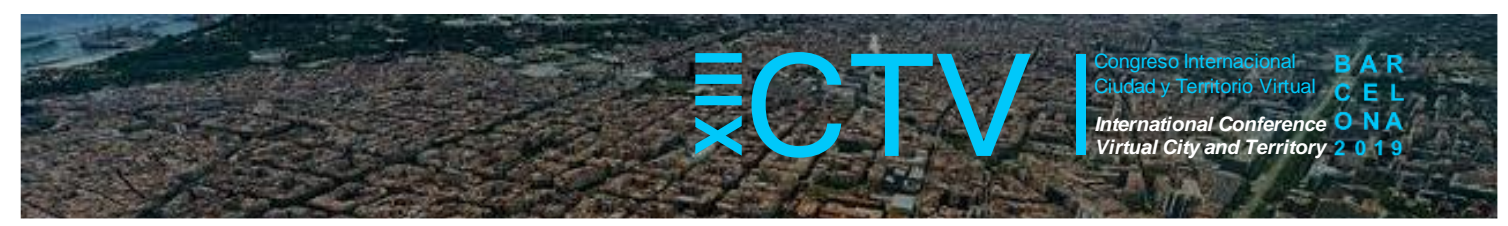

ecosistemas parque Isla Salamanca, Ciénaga de Mallorquín y la Ciénaga Grande de Santa Marta. En cuanto al perfil cualitativo las dimensiones ambiental y económica dan valoraciones entre los rangos de atención inmediata y condición inviable con el $64 \%$ y el $79 \%$ respectivamente. Por otra parte, la dimensión social da una de las valoraciones más altas de todas las UAC, exhibiendo un $64 \%$ de la muestra con indicadores en rangos de riesgo bajo y condición viable, traducido en procesos de investigación y estructuración de sistemas de gestión del riesgo más flexibles, diversos y robustos, en sociedades can mayor nivel de capacitación y mejores niveles de vida, capaces de ofrecer mejor y mayor nivel de respuesta ante la crisis.

La UAC 4-Vertiente Norte de La Sierra Nevada de Santa Marta, presenta impactos fuertes por inmersión y desaparición de una amplia franja de playas destinadas a la industria turística, con efectos colaterales en la infraestructura de soporte. La ciudad de Riohacha en el departamento de la Guajira presenta baja afectación en un escenario de incremento de $1,5^{\circ} \mathrm{C}$; sin embargo, el riesgo combinado de erosión costera y aumento en el nivel del mar, puede representar alto riesgo de afectación. El perfil cualitativo refleja condiciones favorables en la dimensión ambiental, mostrando un $58 \%$ de la muestra evaluada con indicadores en situación de riesgo bajo y condición viable, además de presentar el $0 \%$ de la muestra en condición inviable; el caso opuesto se observa en la dimensión económica, en el que el $83 \%$ de la muestra presenta valoración entre los rangos de condición inviable y riesgo crítico. Lo anterior refleja un sistema potencialmente capaz de absorber impactos desde la dimensión ambiental; no obstante, la gestión de los recursos desde la dimensión económica, no ofrece sustento a iniciativas de gestión del riesgo, lo que sumado a niveles críticos del componente social termina por debilitar el sistema frente a la crisis.

Finalmente, la UAC 5-De la Alta Guajira corresponde al borde costero del departamento de La Guajira, con poca intervención antropogénica por las condiciones socio-culturales y ambientales del territorio. Aquí los municipios costeros de Uribia y Manaure, con crecimiento basado en la explotación del carbón, la sal y la pesca desaparecen prácticamente con el incremento de $1,5^{\circ} \mathrm{C}$. Puede identificarse el grave riesgo de inundación del puerto carbonero de Puerto Bolívar en el municipio Uribia, al igual que la Central de Distribución del Gaseoducto Transoceánico.

El perfil cuantitativo de la UAC 5, presenta dos escenarios opuestos, por una parte, la dimensión ambiental con la mejor valoración de las cinco UAC, la cual presenta el $73 \%$ con indicadores en condición viable y, por otra parte, la dimensión económica con la valoración más baja de todas, exhibiendo un $100 \%$ con indicadores en rangos de riesgo alto y condición inviable. La UAC de la Guajira, presenta la valoración de la gestión ambiental favorable; sin embargo, la gestión de las dimensiones social y económica reflejan escenarios críticos ante cualquier riesgo, con ausencia de valores positivos en manejo de recursos económicos y del componente social, lo que hace inflexible y frágil la respuesta de gestión del riesgo.

\section{Conclusiones}

En definitiva, el panorama para las principales ciudades del litoral caribe colombiano exhibe un nivel mínimo de tolerancia (ver figura 1) en los plazos para el emprendimiento de serios programas de gestión del riesgo por ANM, siendo la ciudad de Cartagena en el departamento de Bolívar un caso crítico, en el que puede pensarse incluso en el traslado gradual de los 


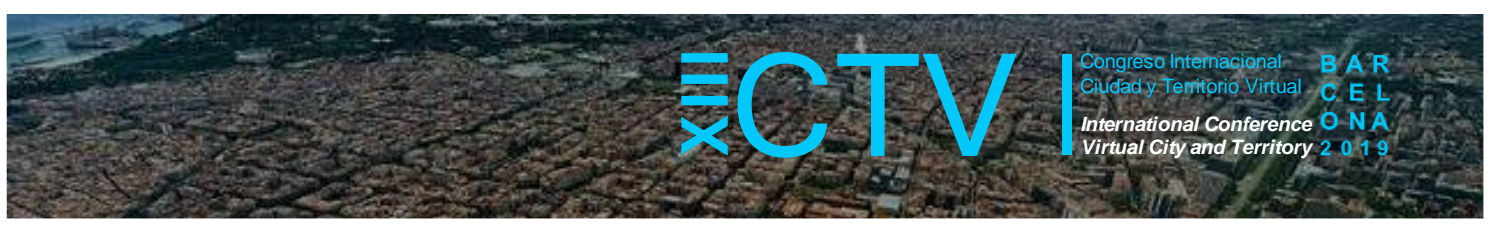

habitantes e infraestructura dispuesta sobre la franja costera a día de hoy, como única estrategia para la reducción del riesgo. Es necesario acotar que, si bien las ciudades como Barranquilla, Santa marta y Cartagena han consolidado un importante nivel de industria, infraestructura portuaria y de servicios con grandes aportes a la economía local y nacional, el nivel de inversión en la planificación urbana, gestión de los recursos ambientales y mejoramiento de la calidad de vida ha sido escaso, en algunos casos mínimo como en La Guajira; traduciéndose en sistemas frágiles y con baja resiliencia urbana aun cuando sean comunidades culturalmente resilientes.

Figura 1. Afectación por ANM en Unidades Ambientales Costeras 1 a 5



Fuente: Elaboración a partir de información de Surgin Seas - Google Earth - DANE - DNP-IDEAM

Agradecimientos: Se hace un reconocimiento especial a la Universidad del Norte por apoyar al estudiante Jim Hurtado de la Maestría en Urbanismo y Desarrollo Territorial.

Contribuciones de los autores: El primer autor ha desarrollado la construcción teórica y la orientación metodológica, el segundo autor ha desarrollado la contrastación cartográfica y la interpretación de datos. Ambos autores han consolidado la presente investigación.

Conflicto de Intereses: Los autores declaran que no hay conflicto de intereses. 


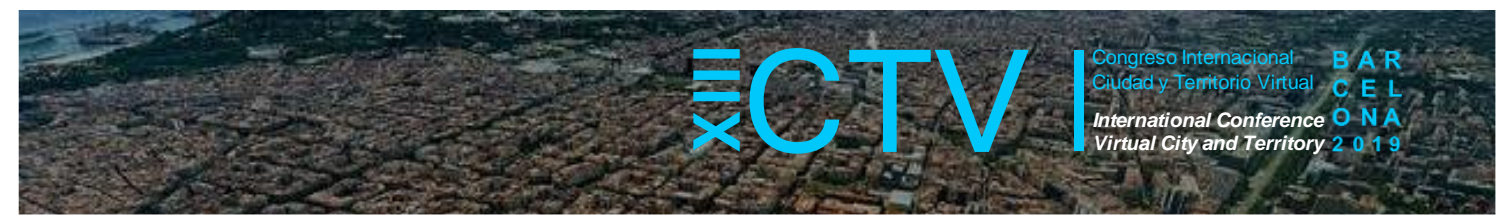

\section{Bibliografía}

ARUP. (2011). Water resilience for cities. Helping cities build water resilience today, to mitigate the risks of climate change tomorrow. Recuperado de https://www.arup.com/perspectives/publications/research/section/urban-life-water-resilience-forcities?query=resilience

Arup Group. (2019). Facing up to the future: The City Resilience Index. Recuperado de https://www.arup.com/perspectives/city-resilience-index

Bedoya, M., Benavides, H., Cabrera, M., Carrillo, H., Ceballos, J. L., Contreras, C., .. . Tobón, E. (2010). Capítulo Cuatro Vulnerabilidad. In M. Cabrera, M. Duarte, M. M. Gutierrez, \& P. Lamprea (Eds.), Segunda comunicación nacional ante la convención marco de las naciones unidas sobre cambio climático (pp. 193-320). Bogotá, Colombia: Instituto de Hidrología, meterología y estudios ambientales - Ideam. 2019. Recuperado de http://www.minambiente.gov.co/index.php/comunicaciones-nacionales-de-cambioclimatico/segunda-comunicacion\#documentos

CLIMATE CENTRAL. (2019). Recuperado de https://www.climatecentral.org. https://seeing.climatecentral.org/\#12/10.4026/-

75.4762? show=lockinAnimated\&level=2\&unit=feet\&pois=hide

Córdoba Larrarte, C. F., Ocampo Niño, J. C., \& Villalba, D. C. (2015). Debilidad institucional de las contralorías departamentales según índice de transparencia de entidades públicas - ITEP. Bogotá, D.C.: Auditoría General de la República. Recuperado de http://www.auditoria.gov.co/Biblioteca documental/Publicaciones/AGRip-dt-09.pdf

DANE. (2009). COLECCIÓN DOCUMENTOS - ACTUALIZACIÓN 2009. Bogotá, D.C: Recuperado de https://www.dane.gov.co/files/investigaciones/fichas/Deficit vivienda.pdf

Deal, B., Pan, H., Pallathucheril, V., \& Fulton, G. (2017). Urban Resilience and Planning Support Systems: The Need for Sentience. Journal of Urban Technology, 24(1), 29-45. DOI: https://doi.org/10.1080/10630732.2017.1285018

Departamento Administrativo Nacional de Estadística - DANE. (2019). Geoportal DANE. Recuperado de https://geoportal.dane.gov.co

Departamento Administrativo Nacional de Estadística DANE. (2019). Sistema Estadístico Nacional. Recuperado de https://www.dane.gov.co/index.php/sistema-estadistico-nacional-sen

Departamento Nacional de Planeación - DNP. (2018). Índice Municipal de Riesgo de Desastres Ajustado por Capacidades. Bogotá, D.C.: Departamento Nacional de Planeación. Recuperado de https://colaboracion.dnp.gov.co/CDT/Prensa/IndiceMunicipaldeRiesgodeDesastres.pdf

Departamento Nacional de Planeación - DNP. (2019). Observatorio del Sistema de Ciudades. Recuperado de https://osc.dnp.gov.co

Departamento Nacional de Planeación - DNP. (2019). Terridata. Recuperado de https://terridata.dnp.gov.co

División de Población de la Comisión Económica para América Latina y el Caribe-CEPAL Centro Latinoamericano y Caribeño de Demografía-CELADE. (2018). Proyecto de primer informe regional sobre la implementación del Consenso de Montevideo sobre Población y Desarrollo. Santiago: Naciones Unidas. Recuperado de https://repositorio.cepal.org/bitstream/handle/11362/43708/1/S1800378 es.pdf 


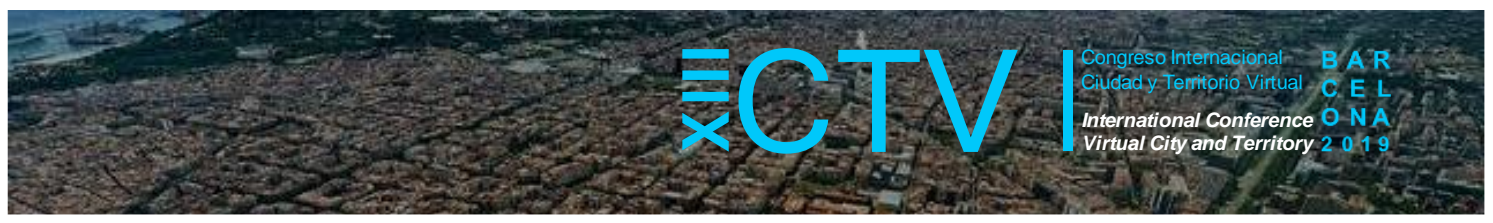

DNP. (2019). Fichas y tableros territoriales. Recuperado de https://terridata.dnp.gov.co/\#/perfiles

IDEAM - Instituto de Hidrología, Meteorología y Estudios Ambientales. (2019). IDEAM - Instituto de Hidrología, Meteorología y Estudios Ambientales. Recuperado de http://www.ideam.gov.co

IDEAM, PNUD, MADS, DNP, CANCILLERÍA. (2017). Análisis de vulnerabilidad y riesgo por cambio climático en Colombia. Tercera Comunicación Nacional de Cambio Climático. Bogotá D.C., Colombia.: IDEAM, PNUD, MADS, DNP, CANCILLERÍA, FMAM.

Instituto Nacional de Salud. (2007). FICHAS TÉCNICAS INDICADORES DE AGUA. Bogotá, D.C. Recuperado de https://www.procuraduria.gov.co/portal/media/file/38.pdf

Intergovernmental Panel on Climate Change - IPCC. (2019). IPCC Special Report on Climate Change, Desertification, Land Degradation, Sustainable Land Management, Food Security, and Greenhouse gas fluxes in Terrestrial Ecosystems. Recuperado de https://www.ipcc.ch/site/assets/uploads/2019/08/Fullreport-1.pdf

Leitner, H., Sheppard, E., Webber, S., \& Colven, E. (2018). Globalizing urban resilience. Urban Geography, 39(8), 1276-1284. DOI: https://doi.org/10.1080/02723638.2018.1446870

Meerow, S., \& Newell, J. (2019). Urban resilience for whom, what, when, where, and why? Urban Geography, 40(3), 309-329. DOI: https://doi.org/10.1080/02723638.2016.1206395

Méndez Gutiérrez del Valle, R. (2012). Ciudades y metáforas: sobre el concepto de resiliencia urbana. Ciudad y territorio: Estudios territoriales (172), 2015-232. Recuperado de https://www.fomento.gob.es/arquitectura-vivienda-y-suelo/urbanismo-y-politica-de-

suelo/estudios-y-publicaciones/revista-ciudad-y-territorio-estudios-territoriales

MINISTERIO DE EDUCACIÓN NACIONAL. (2014). Sistema nacional de indicadores educativos para los niveles de preescolar, básica y media en Colombia. Recuperado de https://www.mineducacion.gov.co/1621/articles-

329021 archivo pdf indicadores educativos enero 2014.pdf

Ministerio de Salud y Protección Social. (2019). Cifras de aseguramiento en salud. Recuperado de https://www.minsalud.gov.co/proteccionsocial/Paginas/cifras-aseguramiento-salud.aspx

Ministerio de Salud y Protección Social. (2019). Sistema Integrado de la Información de la Protección Social. Recuperado de https://sig.sispro.gov.co/sigmsp/index.html

Naciones Unidas. (2019). Naciones Unidas. Objetivos del Desarrollo Sostenible. Recuperado de https://www.un.org/sustainabledevelopment/es/

Naciones Unidas. (2019). Naciones Unidas, Construyendo un Futuro Juntos. Cambio Climático. Recuperado de https://www.un.org/es/sections/issues-depth/climate-change/index.html

Næss, P., Saglie, I.-L., \& Richardson, T. (2019). Urban sustainability: is densification sufficient? European Planning Studies, 1-20. DOI: https://doi.org/10.1080/09654313.2019.1604633

Narula, K. (2014). Climate Change and Oceans - Growing Threat and Implications for India. Maritime Affairs: Journal of the National Maritime Foundation of India, 10(2), 96-119. DOI https://doi.org/10.1080/09733159.2014.969550

Oficina de las Naciones Unidas para la Reducción del Riesgo de Desastres - UNISDR. (2012). Cómo desarrollar ciudades más resilientes - Un Manual para alcaldes y líderes del gobierno local. Ginerbra, Suiza: Naciones Unidas. Recuperado de https://www.unisdr.org/files/26462 manualparalideresdelosgobiernosloca.pdf 




Oficina de las Naciones Unidas para la Reducción del Riesgo de Desastres-UNDRR. (2019). Desarrollando Ciudades Resilientes: ¡Mi ciudad se está preparando! Recuperado de https://www.eird.org/camp-10-15/10-esenciales.html

ONU HABITAT. (2018). CITY RESILIENCE PROFILING TOOL. BARCELONA, ESPAÑA: ONU HABITAT. Recuperado de http://urbanresiliencehub.org/wp-content/uploads/2018/02/CRPTGuide.pdf

Oviedo Torres, B. E. (2010). GENERACIÓN DE ESCENARIOS DE CAMBIO CLIMÁTICO REGIONALES Y LOCALES A PARTIR DE MODELOS GLOBALES - GUÍA PARA TOMADORES DE DECISIONES. Bogotá, Colombia: Instituto de Hidrología, Meteorología y Estudios Ambientales - IDEAM. Recuperado de http://www.ideam.gov.co/documents/21021/21138/Gu\%C3\%ADa+Escenarios+para+Tomadore s+de+Decisiones.pdf/fa7abe38-43cc-49c8-96a3-f2b5c24ecce3

Pedersen Zari, M. (2015). Ecosystem processes for biomimetic architectural. Architectural Science Review, 58(2), 106-119. DOI: https://doi.org/10.1080/00038628.2014.968086

Programa de la Naciones Unidas para el Desarrollo - PNUD. (2007). Informe sobre Desarrollo Humano 2007-2008 - La lucha contra el cambio climático: Solidaridad frente a un mundo dividido. Madrid: Grupo Mundi-Prensa. Recuperado de http://hdr.undp.org/sites/default/files/hdr 20072008 summary spanish.pdf

Rubiera Morollón, F., González Marroquin, V. M., \& Pérez Rivero, J. L. (2016). Urban sprawl in Spain: differences among cities and causes. European Planning Studies, 24(1), 207-226. DOI: https://doi.org/10.1080/09654313.2015.1080230

Rumbach, A. (2017). At the roots of urban disasters: Planning and uneven geographies of risk in Kolkata, India. Journal of Urban Affairs, 39(6), 783-799. DOI: https://doi.org/10.1080/07352166.2017.1282771

Servicio Geológico de Colombia - SGC. (2019, 6 1). Servicio Geológico Colombiano. Recuperado de https://srvags.sgc.gov.co/Flexviewer/Estado Cartografia Geologica/

Sherrieb, K., Norris, F., \& Galea, S. (2010). Measuring Capacities for Community Resilience. Social Indicators Research, 99(2), 227-247. DOI: https://doi.org/10.1007/s11205-010-9576-9

Stead, D. (2014). Urban planning, water management and climate change strategies: adaptation, mitigation and resilience narratives in the Netherlands. International Journal of Sustainable Development \& World Ecology, 21(1), 15-27. DOI: https://doi.org/10.1080/13504509.2013.824928

UN-Habitat. (2018). Guide to the City Resilience Profiling Tool. An introductory guide to the City Resilience Profiling Tool. Recuperado de http://urbanresiliencehub.org/wpcontent/uploads/2018/10/CRPT-Guide-Pages-Online.pdf

United Nations Development Programme - UNDP. (2014). Human Development Report 2014. Washington DC: Communications Development Incorporated. Recuperado de http://hdr.undp.org/sites/default/files/hdr14-report-en-1.pdf

Zhai, G., Li, S., \& Chen, J. (2015). Reducing Urban Disaster Risk by Improving Resilience in China from a Planning Perspective. Human and Ecological Risk Assessment: An International Journal, 21(5), 1206-1217. DOI: https://doi.org/10.1080/10807039.2014.955385 